\title{
Loop Quantum Gravity and the Very Early Universe
}

\author{
Abhay Ashtekar*† \\ Institute for Gravitational Physics and Geometry, Physics Department, Penn State, University \\ Park, PA 16802, U.S.A. \\ E-mail: ashtekar@gravity.psu.edu
}

This brief overview is addressed to beginning researchers. It begins with a discussion of the distinguishing features of loop quantum gravity, then illustrates the type of issues that must be faced by any quantum gravity theory, and concludes with a brief summary of the status of these issues in loop quantum gravity. For concreteness the emphasis is on cosmology. Since several introductory as well as detailed reviews of loop quantum gravity are already available in the literature, the focus is on explaining the overall viewpoint and providing a short bibliography to introduce the interested reader to literature.

7th Conference Mathematical Methods in Physics - Londrina 2012,

16 to 20 April 2012

Rio de Janeiro, Brazil

* Speaker.

${ }^{\dagger}$ A footnote may follow. 
Loop quantum gravity (LQG) is a non-perturbative approach aimed at unifying general relativity and quantum physics. Its distinguishing features are: i) Background independence, and, ii) Underlying unity of the mathematical framework used to describe gravity and the other three basic forces of nature. It is background independent in the sense that there are no background fields, not even a space-time metric. All fields - whether they represent matter constituents, or gauge fields that mediate fundamental forces between them, or space- time geometry itself- are treated on the same footing. As in general relativity, they are all dynamical. Moreover, one does not start with a classical space-time metric and then add quantum corrections to geometry in small steps, treating them as perturbations. Rather, all fields, including space-time geometry are quantum mechanical 'from birth'. Finally, while one maintains the deep duality between geometry and gravity at the foundation of general relativity, the basic dynamical variables are spin connections rather than metrics. The metric arises as a composite field constructed from the 'electric fields' that are canonically conjugate to the spin connection. Since the electroweak and strong interactions are also mediated by connections, one now has an uniform mathematical framework to describe all four fundamental forces of Nature. But the primary focus is on gravity: While the quantum nature of space-time has deep influence on dynamics of all other fields especially in the Planck regime, in contrast to string theory, one does not attempt to unify all forces of Nature. In this respect, the strategy is analogous to the one used in QCD rather than the one that drove grand unified theories.

In this brief overview I will use loop quantum cosmology (LQC) to illustrate the main ideas. ${ }^{1}$ The main advantage of this strategy is that students and other beginning researchers can grasp the goals, challenges, key ideas, advances and limitations in a rather simple setting. The main drawback is that this approach leaves out some of the key advances such as the understanding of quantum horizons through quantum geometry, the issue of information loss, and spin foams, especially the recent calculations of the graviton propagators in the non-perturbative setting of LQG [7, 8, 9]. For the most recent overviews of the key ideas in these and other topics, see review articles in Ref. $[10,11,12,13]$ that will appear in the proceedings of the the third Zakopane school on quantum geometry and quantum gravity.

Keeping this limitation in mind, let us begin with a list of some of the long standing conceptual and technical issues which must be faced by any quantum theory of gravity. In general relativity, gravity is encoded in the very geometry of space-time. The most dramatic features of general relativity can be traced back to this dual role of geometry: the expansion of the universe, the big-bang, formation of black holes and emergence of gravitational waves as ripples of space-time curvature. Already in the classical theory, it took physicists several decades to truly appreciate the dynamical nature of geometry and to get used to the absence of a kinematic background geometry. In quantum gravity, this paradigm shift leads to a new level of difficulties. ${ }^{2}$

- The absence of a background geometry implies that classical dynamics is generated by con-

\footnotetext{
${ }^{1}$ For more detailed treatments, the following reviews should be helpful to the beginning researchers. A short summary of the history of quantum gravity can be found in [1] and a detailed discussion of conceptual problems in [2]. A brief mathematical introduction to loop quantum gravity can be found in [3] and much more detailed treatments in $[4,5,6]$.

${ }^{2}$ There is a significant body of literature on this issue; see e.g., [2] and references therein. These difficulties are now being discussed also in the string theory literature in the context of the AdS/CFT conjecture.
} 
straint equations. In the quantum theory, physical states are solutions to quantum constraints. All of physics, including the dynamical content of the theory, has to be extracted from these solutions. But there is no external time to phrase questions about evolution. Therefore we are led to ask: Can we extract, from the arguments of the wave function, one variable which can serve as emergent time with respect to which the other arguments 'evolve'? If not, how does one interpret the framework? What are the physical (i.e., Dirac) observables? In a pioneering work, DeWitt proposed that the determinant of the 3-metric can be used as an 'internal' time [14]. Consequently, in much of the literature on the Wheeler-DeWitt (WDW) approach to quantum cosmology, the scale factor is assumed to play the role of time, although often only implicitly. However, in closed models the scale factor fails to be monotonic due to classical recollapse and cannot serve as a global time variable already in the classical theory. Are there better alternatives at least in the simple setting of quantum cosmology?

- Can one construct a framework that cures the short-distance difficulties faced by the classical theory near singularities, while maintaining an agreement with it at large scales? By their very construction, perturbative and effective descriptions have no problem with the second requirement. However, physically their implications can not be trusted at the Planck scale and mathematically they generally fail to provide a deterministic evolution across the putative singularity. In LQG the situation is just the opposite. Since the emphasis is on background independence and non-perturbative methods, a priori it is not clear whether the theory also has a rich semi-classical sector. Do the novel dynamical corrections unleashed by the underlying quantum geometry naturally fade away at macroscopic distances or do they have unforeseen implications that prevent the theory from reproducing general relativity at large scales? Some of these unforeseen problems are discussed in, e.g., [15, 16, 17].

Next, the dual role of the space-time metric also implies that space-time itself ends when the gravitational field becomes infinite. This is in striking contrast with Minkowskian physics, where singularity of one specific field has no bearing at all on the space-time structure or on the rest of physics. In general relativity, singularities of the gravitational field represent an absolute boundary of space-time where all of physics comes to a halt.

Now, it is widely believed that the prediction of a singularity — such as the big-bang - in classical general relativity is primarily a signal that the theory has been pushed beyond the domain of its validity and cannot therefore be trusted. One needs a quantum theory of gravity to analyze true physics. This expectation immediately leads to a host of questions which have been with us for several decades now:

- How close to the big-bang does a smooth space-time of GR make sense? Inflationary scenarios, for example, are based on a space-time continuum. Can one show from some first principles that this is a safe approximation already at the onset of inflation?

- Is the Big-Bang singularity naturally resolved by quantum gravity? This possibility led to the development of the field of quantum cosmology in the late 1960s. The basic idea can 
be illustrated using an analogy to the theory of the hydrogen atom. In classical electrodynamics the ground state energy of this system is unbounded below. Furthermore, because an accelerating electron radiates continuously, it must fall into the proton making the atom extremely unstable. Quantum physics intervenes and, thanks to a non-zero Planck's constant, the ground state energy is lifted to a finite value, $-m e^{4} / 2 \hbar^{2} \approx-13.6 \mathrm{ev}$. The hope was that a similar mechanism would resolve the big-bang and big crunch singularities in simple cosmological models. Can the quantum nature of geometry underlying LQG naturally lead to such a resolution?

- Is a new principle/ boundary condition at the big bang or the big crunch essential to provide a deterministic evolution? The most well known example of such a boundary condition is the 'no boundary proposal' of Hartle and Hawking [18]. Or, do quantum Einstein equations suffice by themselves even at the classical singularities?

- Do quantum dynamical equations remain well-behaved even at these singularities? If so, do they continue to provide a deterministic evolution? The idea that there was a pre-bigbang branch to our universe has been advocated by several approaches, most notably by the pre-big-bang scenario in string theory [19] and ekpyrotic and cyclic models inspired by brane world ideas [20,21]. However, in such perturbative treatments there is always a smooth continuum in the background and hence the dynamical equations break down at the singularity. Consequently, the pre-big-bang branch is not joined to the current post-big-bang branch by a deterministic evolution. The hope has been that non-perturbative effects would remedy this situation in the future. LQG, on the other hand, does not have a continuum geometry in the background and the treatment is non-perturbative. So, one is led to ask: Can the LQC quantum Einstein's equation provide a deterministic evolution across the big bang and the big-crunch?

- If there is a deterministic evolution, what is on the 'other side'? Is there just a quantum foam from which the current post-big-bang-branch is born, say a 'Planck time after the putative big-bang'? Or, was there another classical universe as in the pre-big-bang and cyclic scenarios, joined to ours by deterministic equations?

These are some of the central, foundational questions that must be addressed by any theory of quantum gravity. In addition, there are important phenomenological issues. The early universe offers us the best chance to confront quantum gravity theories with observations. The past two decades have witnessed a transformation of cosmology to precision science, thanks to the spectacular success of space missions, COBE and WMAP. These missions have provided a rather detailed snapshot of the universe at the surface of last scattering which shows that the universe was then extremely homogeneous, with tiny inhomogeneities of one part in $10^{5}$. But these very tiny fluctuations are responsible for the observed large scale cosmic structure. More precisely, the CMB inhomogeneities have been taken as initial conditions to carry out numerical simulations using classical gravity and standard (astro)physics. They reproduce qualitative features of the observed large structure of the universe. Thus the origin of the observed cosmic structure lies in the tiny $C M B$ inhomogeneities. 
For theories of the early universe perhaps the most interesting consequence of the improved observations to date is that they have narrowed down the theoretical scenarios for structure formation. Inflation has now emerged as the leading candidate to successfully account for the CMB inhomogeneities and therefore of the cosmic structure. Therefore, although the criticisms raised, especially by the general relativity community, against the motivations that were originally given to advocate an inflationary phase are valid, one can now choose to forego those motivations and focus just on the success of the inflationary paradigm in explaining the CMB inhomogeneities. From this standpoint, the inflationary scenario pushes back the issue of the origin of the cosmic structure from the CMB era when the matter density was only $\sim 10^{-114} \rho_{\mathrm{Pl}}$ to the onset of the slow roll phase of inflation when the mater density was about $10^{-11} \rho_{\mathrm{Pl}}$. However, the inflationary scenario assumes that space-time is described by general relativity, with its big bang singularity. Therefore, one is led to ask:

- If the singularity can be resolved, can one extend our current understanding of the origin of structure, even further, all the way to the true beginning? Can one set natural initial conditions there? Can one construct a theoretical framework to evolve these initial conditions through the Planck era where general relativity is replaced by an appropriate quantum theory of space-time geometry? This would require an extension of the quantum field theory on curved space-times to that on quantum geometry. If one can, could one systematically evolve the initial conditions from the Planck era over the 11 orders of magnitude in density and curvature to the epoch when inflation began? If so, would the power spectrum at the end of inflation agree with that predicted by standard inflation? Such an extension will move the reach of observational cosmology all the way to the Planck scale, thereby enabling a confrontation of quantum gravity theories with observations.

over the past 6 years or so, these and related questions have been addressed in detail through $L Q C$. (For a detailed review, see [22].) I will conclude with a brief summary of the results and their implications to the issues raised above.

First, in the cosmological setting, one can 'deparameterize' the quantum Hamiltonian constraint of general relativity. At a fundamental level, the constraint simply restricts the wave functions which are to be regraded as physical quantum states. But it can be 'deparameterized' in the sense that one can single out one of the dynamical variable as the time parameter —or internal clock - and coherently interpret the quantum constraint equation as the evolution equation in this internal time. This is necessary and natural because, since the quantum state described a probability amplitude for various space-time geometries to occur, the familiar notions of proper or conformal time that are commonly used in cosmology no longer have a clear-cut meaning. Instead, one can use a matter variable, typically a scalar field as the 'clock' with respect to which physical quantities of interest -such as curvature, shears, anisotropies, matter density and pressure- evolve. This corresponds to adopting a 'relational time' a la Leibniz.

In the details of deparametrization, however, quantum geometry underlying LQG plays a key role. It introduces new and totally unforeseen quantum effects which naturally resolve the cosmological singularities even when one introduces matter fields that satisfy all the standard energy 
conditions. In the cosmological setting under consideration, in general relativity these energy conditions lead to the powerful singularity theorems due to Penrose, Hawking and others. These theorems are bypassed because the left hand side of Einstein's equations is modified by the quantum geometry effects. However, to bring out new physics in familiar terms, in the homogeneous models one can recast these quantum modified equations simply by algebraic manipulations so that the corrections are shifted to the right hand side, i.e., matter side. For definiteness, let us consider the simplest of these, the Friedmann, Lemaitre, Robertson Walker models. The standard Friedmann equation in general relativity is $H^{2}:=(\dot{a} / a)^{2}=(8 \pi G / 3) \rho$, where $H$ is the Hubble parameter, $a$ the scale factor and the 'dot' refers to derivative in proper time. Now, the peak of the quantum wave function that satisfies the Hamiltonian constraint follows an 'effective' trajectory given by

$$
H^{2}:=(\dot{a} / a)^{2}=\frac{8 \pi G}{3} \rho\left(1-\frac{\rho}{\rho_{\mathrm{B}}}\right)
$$

where the term in the last parenthesis represents the quantum correction. There is an analytical formula for $\rho_{\mathrm{B}}$ and its numerical value turns out to be $\rho_{\mathrm{B}} \approx 0.41 \rho_{\mathrm{Pl}}$. In general relativity, $\dot{a}$ cannot vanish if $\rho>0$. If $\dot{a}>0$ we are in the expanding branch; the universe starts out from a big bang and expands out forever. If $\dot{a}<0$ we are in a contracting branch where the universe ends in a big crunch. But because of the quantum correction, when $\rho=\rho_{\mathrm{B}} \dot{a}=0$ and therefore one can now pass smoothly from a contracting branch in the past to an expanding branch in the future, with a quantum bounce at $\rho=\rho_{B}$. Thus the big bang singularity is naturally resolved by quantum gravity corrections. ${ }^{3}$ This resolution have been shown to persist if we add a cosmological constant and/or an inflationary potential, consider spatially closed universes, include anisotropies (Bianchi models) or the simplest type of inhomogeneities and gravitational waves (Gowdy models). In this sense the result is robust. But in more general models, the nature of the bounce is more complicated. in addition to the bounce in the density, there are also other 'bounces'. Roughly, anytime a curvature scalar enters the Planck regime, a quantum correction kicks in, preventing further growth. The physical mechanism lies in a new repulsive force whose origin lies in the quantum nature of geometry. When the space-time curvature (or matter density) approaches the Planck scale, the repulsive force becomes so strong that it overwhelms the classical gravitational attraction a la general relativity and dilutes these quantities, thereby preventing singularities. But a key characteristic of this new force is that it decays rapidly as one moves away from the Planck regime, so that general relativity quickly becomes a good approximation. Because of this key feature, the potential ultraviolet-infrared tension metls away. Finally, this singularity resolution does not require any new boundary conditions: the quantum version of Einstein's equations, derived in the cosmological context, suffices.

This analysis provides a detailed theory of quantum geometry for homogeneous models in the Planck era. Quantum field theory on this quantum geometry has been developed. Very recently, it was applied to extend the inflationary scenario all the way to the Planck regime. The repulsive force that we just discussed has the diluting effect which homogenizes curvature and matter of a scale of approximately $10 \ell_{\mathrm{Pl}}$. Furthermore, if one uses a standard inflationary scenario and goes back in

\footnotetext{
${ }^{3}$ Now, there are also different types of singularity theorems in classical general relativity, due to Borde, Guth and Vilenkin, which apply even when all energy conditions are not met, as in inflationary models. These are bypassed because they assume eternal inflation whereas in LQC there is a contracting phase prior to the bounce.
} 
time all the way to the (density) bounce using effective equations of LQC one finds that the entire observable universe arose due to expansion of a tiny region of less than $10 \ell_{\mathrm{Pl}}$ radius. Therefore, it suffices to assume initial conditions only for these small regions and it is then reasonable to assume that such regions were homogeneous at the bounce except for the unceasing quantum fluctuations that cannot be gotten rid of even in principle. The precise form of these initial conditions is called quantum homogeneity. Equations of quantum field theory on quantum space-times enables on to evolve these initial conditions all the way to the end of inflation. Detailed numerical solutions have shown that the resulting power spectrum is very close to that of standard inflation, and compatible with the 7 year WMAP data. Thus, the genesis of the cosmic structure is pushed back from the inflationary epoch all the way to the Big Bounce, covering some 11 orders of magnitude in matter density and curvature. Finally, there is also a small window in the parameter space where the theory predicts new effects that could be observed in future missions. (For a summary see [22, 23] and for details see the recent and forthcoming papers by Agullo, Ashtekar and Nelson.)

To summarize, LQC has faced many of the long standing difficult problems that any theory of quantum gravity must address in the cosmological context. Results to date are surprising in many respects. The new effects of origin in the Planck scale physics are strong yet subtle. They are strong enough to resolve the singularity of general relativity and yet subtle enough to ensure agreement with general relativity at low curvature. Furthermore, the detailed framework has now advanced sufficiently to make direct contact with phenomenology and observations. Finally, on the conceptual side, these advances in LQC provide hints and tools to further develop loop quantum gravity also outside cosmology.

I hope this overview will serve as an invitation to young researchers to go deeper into LQC and LQG.

Acknowledgments: There are well over a thousand papers in loop quantum cosmology and I have profited a great deal from discussions with the authors of a large number of them. Of course I could not do justice to all the work in this limited space; I hope I have managed to convey at least a flavor of the results to date. There is inevitable overlap with some of the reviews I have written, particularly [24, 22]. This work was supported in part by the NSF grant PHY-1205388, the Eberly research funds of Penn state.

\section{References}

[1] A. Ashtekar, Gravity and the Quantum, New Journal of Physics 7 (2005) 200-232 [gr-qc/0410054].

[2] A. Ashtekar and J. Stachel, Conceptual problems of Quantum Gravity, Birkhäuser, Boston, 1988.

[3] A. Ashtekar and J. Lewandowski, Quantum geometry and its applications, Encyclopedia of Mathematical Physics, Elsevier (2006) 230-236; also available at http: www.gravity.psu.edu/poparticles.

[4] A. Ashtekar and J. Lewandowski, Background independent quantum gravity: A status report, Class. Quant. Grav. 21 (2004) R53-R152 [gr-qc/0404018].

[5] C. Rovelli Quantum Gravity, CUP, Cambridge, 2004. 
[6] T. Thiemann, Introduction to Modern Canonical Quantum General Relativity, CUP, Cambridge, at press.

[7] C. Rovelli, Graviton propagator from background-independent quantum gravity, Phys. Rev. Lett. 97 (2006) 151301 [gr-qc/0508124].

[8] E. Bianchi, L. Modesto, C. Rovelli and S. Speziale, Graviton propagator in loop quantum gravity, Class. Quantum Grav. 23 (2006) 6989-7028 [gr-qc/0604044].

[9] E. R. Livine and S. Speziale, Group Integral Techniques for the Spinfoam Graviton Propagator, gr-qc/0608131.

[10] A. Ashtekar, arXiv:1201.4598.

[11] H. Sahlmann and K. Giesel, arXiv:1203.2733.

[12] F. Barbero, J. Lewandowski and E. Villasenor, arXiv:1203.0174

[13] C. Rovelli, arXiv:1102.3660.

[14] B. S. DeWitt, Quantum Theory of Gravity I. The Canonical Theory, Phys. Rev. 160 (1967) 1113-1148.

[15] D. Green and W. Unruh, Difficulties with recollapsing models in closed isotropic loop quantum cosmology, Phys. Rev. D 70 (2004) 103502 [gr-qc/04-0074].

[16] A. Ashtekar, T. Pawlowski and P. Singh, Quantum nature of the big bang: An analytical and numerical investigation I, Phys. Rev. D 73 (2006) 124038 [gr-qc/0604013].

[17] A. Ashtekar, T. Pawlowski and P. Singh, Quantum nature of the big bang: Improved dynamics, Phys. Rev. D 74 (2006) 084003 [gr-qc/0604013].

[18] J. B. Hartle and S. W. Hawking, Wave Function Of The Universe, Phys. Rev. D 28 (1983) 2960.

[19] M. Gasperini and G. Veneziano, The pre-big bang scenario in string cosmology, Phys. Rept. 373 (2003) 1 [hep-th/0207130].

[20] J. Khoury, B. A. Ovrut, P. J. Steinhardt and N. Turok, The Ekpyrotic Universe: Colliding Branes and the Origin of the Hot Big Bang, Phys.Rev. D 64 (2001) 123522 [hep-th/0103239].

[21] J. Khoury, B. Ovrut, N. Seiberg, P. J. Steinhardt and N. Turok, From Big Crunch to Big Bang, Phys.Rev. D 65 (2002) 086007 [hep-th/0108187].

[22] A. Ashtekar and P. Singh, Loop quantum cosmology: A status report, Class. Quantum Grav. 28 (2011) 213001 [gr-qc/1108.0893].

[23] I. Agullo, A. Ashtekar and W. Nelson, A quantum gravity extension of the inflationary scenario, Phys. Rev. Lett. (at press), gr-qc/1209.1609.

[24] A. Ashtekar, An introduction to loop quantum gravity through cosmology, Nuovo Cimeto 122 (2007) 1-20 [gr-qc/0702030]. 